P169 (continued)

Objective: We explored quality of implementation during a pilot evaluation in spring 2017, using a multiprong approach: principles-based fidelity, attendance, and participant reaction: interest, relevance, comfort level, and satisfaction.

Description: We conducted live observation of a subset of sessions with a check-list of "core concepts". Facilitators answered questions regarding participant engagement and understanding of concepts presented. Participants completed a session evaluation that assessed understanding of core concepts presented (knowledge), and facilitator rapport. We aimed to assess adherence but also other site-specific nuances associated with learning and key outcomes.

Evaluation: Of the 13 participating families (fathers), most attended six sessions. In total, parents completed 109 after-session (one-page) evaluation forms. Most (90\%) indicated class sessions were somewhat or very useful, somewhat or very interesting, and that participants felt comfortable sharing opinions. Most (94\%) of their responses rated facilitators as supportive and as having addressed participants' needs and interests.

Conclusions and Implications: This process evaluation findings illustrate quality implementation assessment. Overall facilitators had high levels of facilitation skills. The principle-based fidelity addressed the necessity, not only to capture adherence, but also whether the program has been able to achieve the desired results. It also provided insight to implementation that allows for adaptability to "real life" situations without loss of effectiveness. However, it called for a highly-skilled evaluator and developing the indicators of that can be broadly used in community settings.

Funding: USDA.

\section{P170 Exploring the Use of Online Learning in Postsecondary Nutrition Education Courses: A Systematic Review}

Amy Spielmaker, BS, RDN, amykspielmaker@gmail.com, Western Oregon University, 345 Monmouth Avenue N, Monmouth, OR 97361; Megan Patton-López, PhD, RDN; Zubaida Qamar, PhD, University of California, San Francisco; Mallory Koenings, PhD, RDN, USDA, National Institute of Food and Agriculture; Brandy-Joe Milliron, PhD, Drexel University; Marissa Burgermaster, PhD, Columbia University

Background (Background, Rationale, Prior Research, and/or Theory): The internet and new information technologies have transformed education. Nutrition education is among many postsecondary subjects being taught using online instructional methods. With increasing demand for flexible coursework to prepare nutrition professionals, educators will benefit from a synthesis of what is known about effective online learning for nutrition education courses.

Objective: To investigate the current use of online instruction in undergraduate and graduate nutrition courses, determine course effectiveness, and identify practical recommendations for educators.

Study Design, Setting, Participants, Intervention: We systematically reviewed studies via a comprehensive search of major databases using a standardized search strategy. Studies selected for inclusion were those that evaluated the knowledge and/or perceptions of undergraduate or graduate students in postsecondary nutrition education courses delivered completely or primarily online. We considered both experimental and epidemiological study designs.

Outcome Measures and Analysis: We extracted data on course instructional design, study design, participants, and outcomes from the studies meeting our inclusion criteria. Four reviewers independently assessed study quality using the Nutrition Evidence Library Bias Elimination Tool. Results: Nineteen of 5,803 search results met the inclusion criteria. Most involved asynchronous online courses in health professional studies (e.g. dietetics, nursing, medicine). Few studies employed an experimental design and many lacked details regarding the course's instructional design. Among studies with a comparison group $(n=7)$, there were no differences in nutrition knowledge between online and face-to-face learners. Results were inconclusive regarding students' perceptions.

Conclusions and Implications: Current reports suggest that neither online nor face-to-face nutrition courses are superior and students' experiences with online learning vary. Recommendations for educators include being engaged with the online class during discussions, providing regular feedback, becoming comfortable with different technologies, and developing course content that facilitates discovery learning. The heterogeneous methods and limited descriptions of instructional design we identified highlight the need for research that elucidates effective approaches to online learning in postsecondary nutrition education courses.

Funding: None.

\section{P171 Field Testing of Complementary Feeding and Nutrition Education Intervention in Selected Philippine Villages}

Julieta B. Dorado, juliedorado2015@gmail.com, Department of Science and Technology_Food and

Nutrition Research Institute, General Santos Avenue, Taguig City, Metro Manila, 01633, Philippines; Clarita R. Magsadia, MS; Rowena V. Viajar, MS; Chona F. Patalen, MS; Glenda P. Azana;

Mario V. Capanzana, PhD

Background (Background, Rationale, Prior Research, and/or Theory): In response to the problem of malnutrition in the Philippine countryside, the Department of Science and Technology, through the Food and Nutrition Research Institute (DOST-FNRI), developed a package of interventions which combines complementary feeding of young children and nutrition education of mothers and caregivers. 